\title{
Relationship Between Xerostomia and Hyposalivation in Senior Chilean People
}

\author{
Cecilia Muñoz ${ }^{1}$, Alejandra Martínez ${ }^{2}$ Maritza Flores $^{3}$, Alfonso Catalán ${ }^{3-4}$
}

\author{
1. Oral Prosthetic Rehabilitation Program, \\ Restorative Department, School of Dentistry, \\ Universidad de Concepción, Concepción, Chile. \\ 2. Oral Prosthetic Rehabilitation Program, \\ Restorative Department, School of Dentistry, \\ Universidad de Concepción, Concepción, Chile. \\ 3. Public Health Department, School of Medicine, \\ Universidad de Concepción, Chile. \\ 4. Removable Prosthetics, Oral Prosthetic \\ Rehabilitation Program Director, Department \\ of Restorative Dentistry, School of Dentistry, \\ Universidad de Concepción, Chile.
}

* Corresponding author: Alfonso Catalán | Universidad de Concepción - Casilla 160-C Concepción, Chile | Phone number: 56-41-2204481 | Fax: 56-41-2207440 | E-mail: acatalan@udec.cl Trabajo recibido el 14/01/2019. Aprobado para su publicación el 05/07/2019

\begin{abstract}
Background: Hyposalivation is an objective decrease in salivary flow and it can produce xerostomia; which is a subjective sensation of dry mouth, common condition in senior population. Objectives: To identify the association between xerostomia and hyposalivation and its risk factors in people aged 60 years and older, and to investigate the association with medications, habits and other oral complications. Methods: 211 participants were included. Xerostomia data was collected using a validated SpanishXerostomia Inventory (XI-sp). Unstimulated whole-salivary flow rates were measured to detect hyposalivation. Results were analyzed using Chi-square, Fisher tests and multivariate logistic regression analysis. $p<.05$. Results: No significant association was detected between Xerostomia and Hyposalivation $(p=.0666)$. Xerostomia $84.3 \%(p=.036)$ and hyposalivation $81.4 \%(p=.004)$ occur more frequently in women. A significant association was found between hyposalivation with the female gender (OR $=2.46,95 \%$ confidence interval $(\mathrm{Cl}): 1.19-5.11, \mathrm{p}=0.015)$; denture stomatitis $(\mathrm{OR}=3.71$, 95\% Cl:1.03-13.3, $p=0.045)$ and atrophic glossitis (OR=3.72, 95\% Cl:1.78-8.1, $\mathrm{p}=0.001)$. Only female gender $(\mathrm{OR}=2.54 ; 95 \% \mathrm{Cl}: 1.19-5.43, \mathrm{p}=0.016)$ was significantly associated with xerostomia. Conclusions: No statistically significant association was found between hyposalivation and xerostomia. A significant association was found between oral candidiasis, denture stomatitis and the atrophic tongue with hyposalivation. Being woman was a risk factor for xerostomia and hyposalivation.

KEY WORDS
\end{abstract}

Xerostomia; Hyposalivation; Risk factors; Medications; Senior people; Alcohol habits.

Rev. Clin. Periodoncia Implantol. Rehabil. Oral Vol. 12(3); 123-126, 2019.

\section{INTRODUCTION}

Currently the world's population and Chilean population are aging, with an increase percentage of senior population; 60 years and more ${ }^{(1,2)}$. Some clinical studies have reported that there is a prevalence between $20 \%$ and $40 \%$ of dry mouth in adults over 50 years' population, concluding that this condition is higher in number in women ${ }^{(3-4)}$.

Hyposalivation corresponds to an objective reduction in salivary flow $^{(3,5-7)}$ while xerostomia is a subjective sensation of dry mouth ${ }^{(5,8-11)}$. It is considered being production of saliva equal to or less than 0.1 or $0.2 \mathrm{~mL}$ per minute ${ }^{(12,13)}$.

Hyposalivation can be produced by several factors, such as dysfunction in salivary gland, autoimmune diseases, other diseases, medications and smoking ${ }^{(3,7,11,14-16)}$. Hyposalivation may or may not be accompanied by the perception of dry mouth or xerostomia, which corresponds to a condition commonly reported in senior people $(3,6,7,9,16,17)$. The prevalence of hyposalivation in general population ranged $14.4 \%$ to $20 \% .18$ Meanwhile in autoimmune diseases is over $50 \%{ }^{(19,20)}$.

The prevalence of xerostomia ranged from $0.9 \%$ to $64.8 \%(11,14,21)$. Researchers adjusted xerostomia-influencing factors such as head and neck radiotherapy, Sjögren syndrome, chemotherapy and Graft-versushost disease ${ }^{(8,10,11,22-24)}$. Other studies relate this condition, in systemic disease, polypharmacy, or simply declare idiopathic $\mathrm{c}^{(3,5,10,15,22,25-27)}$.

Studies have attributed a higher prevalence of oral candidiasis denture stomatitis, atrophic tongue, dysgeusia, fissured tongue, associated with both hyposalivation and xerostomia ${ }^{(3,7,9,18,28)}$. Because of thus, the purpose of this study was to identify the risk factors of xerostomia and hyposalivation in senior people; 60 years and older, and to investigate the association with medication, habits and other oral complications. The hypothesis expected a relationship between Xerostomia and Hyposalivation and at the same time, being women and being senior patients aged 60 years and older. Both were risk factors conditions.

\section{MATERIAL AND METHODOS}

\section{Design}

The study used a cross-sectional non-experimental quantitative research type. The relationship between xerostomia and hyposalivation was evaluated in patients over 60-years old treated at the School of Dentistry at the University of Concepción, Chile.

Patients younger than 60-years old, or people who suffer diseases that do not allow proper examination by the examiner such as psychiatric disorders and bedridden patients, were excluded from the study.

\section{Selection and sample size}

Sampling was randomly conducted, using the existing database of patients in the Diagnostic office, School of Dentistry. The universe of patients 60 years and older who were treated during year 2017 in the School of Dentistry at University of Concepción was 483 people, and the sample calculated for this study was 211 senior people, considering a prevalence of $39.5 \%$, which corresponds to the known prevalence of xerostomia. Data was obtained considering a confidence level of $95 \%$ and a margin of error of $5 \%$, power $80 \%$.

\section{Clinical evaluation}

A medical history was obtained from all patients, including information related to current systemic diseases, regularly use of medication, tobacco smoking, and alcohol consumption. Complete extraoral and intraoral clinical examinations were performed for all patients to detect oral mucosal lesions using Oral Health Survey: Basic Methods,29 as well as and others disorder such as dry mouth, fissure tongue, atrophic glossitis, dysgeusia, and burning mouth syndrome (BMS). The clinical examinations were carried out by a single operator, (AM).

\section{Xerostomia Inventory- Spanish (XI-sp)}

This is a multidimensional survey for measuring dry mouth, which approach evaluates experiential and behavioral aspects of the disease, allowing the relation of results between dry mouth with the levels of salivary secretion of the patient, getting a diagnosis and tracking changes in symptomatology ${ }^{(21,30)}$. The questionnaire was validated to the Spanish ${ }^{(30)}$ and consists of 11 items in which the patient answers "never", "rarely", "occasionally", "often" and "very often". With a score ranging from one to five according to code selected response, delivers a sum between 11 and 55 points, which determines the severity of xerostomia perceived by the 
patient. It was considered that patients did not present xerostomia when they answered "never" to all or most of the questions, that is, when they obtained total values lower than 14.5 points in the xerostomia inventory. The questionnaires were carried out by a single examiner.

\section{Spitting method}

For the unstimulated saliva collection, people were told not to eat, drink, smoke, brush their teeth or put anything into their mouths during the 90 min prior to the exam ${ }^{(31)}$. The samples were taken during the morning between 09:00 to $12: 00 \mathrm{~h}$ due to circadian cycle regulating salivary flow. 12 The flow collection must be performed in a quiet environment with the person sitting in a vertical position, head leaning forwards and open eyes, avoiding any body or oral-facial movements.

Patients are asked to remain still, swallow saliva at the beginning and allowed to produce saliva the following five minutes to passively drain rover the lower lip and fall into a previously calibrated glass tube equipped with a funnel. At the end of the test time patients are required to eliminate the rest of the saliva in the mouth spitting it into the glass tube. The accepted range of normal flow for unstimulated saliva is something above 0.3 to $0.4 \mathrm{~mL} / \mathrm{min}$ during observation ${ }^{(12,31)}$. Flow rate below $0.2 \mathrm{~mL} / \mathrm{min}$ is considered hyposalivation ${ }^{(13,32)}$.

Informed consents were obtained from all patients and this study was approved by the Ethics Committee of the University of Concepción.

\section{Statistical analysis}

Data was analyzed using statistical software (SPSS for Windows [Microsoft] $\vee$ 16.0; SPSS Inc). Values were normally distributed as verified by Kolmogorov-Smirnov test. Nonparametric statistical tests Chisquare test with Yates's correction and Fisher's exact test were used. A multivariate logistic regression models were constructed after checking for collinearity and forward stepwise selection determined the final model. Differences were considered statistically significant when $p<.05$.

\section{RESULTS}

A total of 211 participants were included. There were 159 women and 52 men $(75.4 \%$ and $24.6 \%$, respectively). The average age of the study population was $69.2 \pm 7,1$ years; ranged between 60 and 94 -years old. From all participants, $171(81 \%)$ presented xerostomia and $140(66.4 \%)$ had hyposalivation. Among the 171 patients who reported having xerostomia, 118 of them had hyposalivation, no significant association was detected between xerostomia and hyposalivation $(p=.0666)$. (Table 1).

Table 1: Frequency of patients with xerostomia and hyposalivation.

\begin{tabular}{l|c|c|c|c|c}
\hline \multicolumn{7}{c}{ Xerostomia } \\
\hline \multicolumn{7}{c}{ No Patients } \\
\hline \multicolumn{7}{c}{ NO } & SI & Total & p-value \\
\hline Hyposalivation & SI & 22 & 118 & 140 & 0.06661 \\
\hline No Patients & NO & 18 & 53 & 71 & \\
\hline & Total & 40 & 171 & 211 & \\
\hline
\end{tabular}

Hyposalivation was defined as an unstimulated salivary flow rate of less than 0.2 milliliter per minute. Xerostomia was defined as a score greater than 14,5 total points in the Xerostomia Inventory.

Significant associations were considered with a $p<0.05$

Xerostomia occurs more often in female; 134 (84.3\%), with a statistically significant difference when comparing to male $(p=.036)$. On the other hand, the hyposalivation was significantly higher for women $(p=.004)$, as demonstrated in Table 2.

The medical characteristics of patients showed that hypertension $(58 \%)$ and diabetes $(20,8 \%)$ were the most frequent systemic diseases. Regarding to consumption of prescribed medications, among 211 participants, 51 patients (24. 2\%) did not consume any medication. One hundred-thirty-three participants $(63 \%)$ used between one to three drugs and 27 participants (12.8\%) used four to six different medicine. Among the most commonly drugs used were antihypertensives, antidepressants, anxiolytics, diuretics and anti-diabetics. When comparing drug intake with xerostomia or hyposalivation, a significant relationship only with xerostomia and intake of anxiolytics $(p=.037)$ and tricyclic antidepressants $(p=.038)$ were found, (Table 2)

About $53(25.12 \%)$ of the patients revealed drinking alcohol. While 28 patients (13.27\%) admitted being smokers. A Chi-square test revealed a significant association between smoking habits and hyposalivation $(p=.046)$.
Table 2: Hyposalivation and xerostomia relationship with gender and medications.

\begin{tabular}{|c|c|c|c|c|}
\hline \multirow[b]{2}{*}{ Variable } & \multicolumn{2}{|c|}{ Hyposalivation } & \multicolumn{2}{|c|}{ Xerostomia } \\
\hline & $\begin{array}{c}\mathbf{N}^{\circ} \\
\text { Patients }\end{array}$ & p-value & $\begin{array}{c}\mathbf{N}^{\circ} \\
\text { Patients }\end{array}$ & p-value \\
\hline \multicolumn{5}{|l|}{ Gender } \\
\hline Male & $26 / 140$ & & $37 / 171$ & \\
\hline & & 0.004 & & 0.036 \\
\hline Female & $114 / 140$ & & $134 / 171$ & \\
\hline \multicolumn{5}{|c|}{ Medication } \\
\hline Anxiolytics & & & $6 / 171$ & 0.037 \\
\hline \multicolumn{3}{|c|}{ Antidepressants } & $15 / 171$ & 0.038 \\
\hline
\end{tabular}

Hyposalivation was defined as an unstimulated salivary flow rate of less than 0.2 milliliter per minute. Xerostomia was defined as a score greater than 14,5 total points in the Xerostomia Inventory.

\section{Significant associations were considered with a $p<0.05$}

The relation of hyposalivation and oral mucosal lesions are presented in Table 3. The Chi-square test showed that hyposalivation had a significant association with dysgeusia $(p=.035)$, fissured tongue $(p<.0001)$, atrophic tongue $(p<.0001)$, and denture stomatitis $(p=.005)$. When comparing xerostomia and oral diseases a statistically significant correlation was found between atrophic tongue $(p<.0001)$, and denture stomatitis $(p=$ .005) (Table 4).

Table 3: Relation between oral conditions and hyposalivation.

\begin{tabular}{c|c|c|c}
\hline \multirow{2}{*}{$\begin{array}{c}\text { Oral conditions } \\
\text { and diseases }\end{array}$} & \multicolumn{3}{c}{$\begin{array}{c}\text { Hyposalivation } \\
\mathbf{N}^{\circ} \text { Patients }\end{array}$} \\
\cline { 2 - 4 } & $\mathbf{S I}$ & NO & $\boldsymbol{p}$-Value \\
\hline Dysgeusia & $15 / 139$ & $2 / 71$ & 0.035 \\
\hline Fissure tongue & $42 / 138$ & $6 / 68$ & $<0.0001$ \\
\hline Atrophic glossitis & $59 / 139$ & $11 / 68$ & $<0.0001$ \\
\hline Denture & $27 / 140$ & $4 / 71$ & 0.005 \\
\hline stomatitis & &
\end{tabular}

Hyposalivation was defined as an unstimulated salivary flow rate of less than 0.2 milliliter per minute.

Significant associations were considered with a $p<0.05$

Table 4: Relationship between oral conditions and lesions with xerostomia.

\begin{tabular}{cc|c|c}
\hline \multirow{2}{*}{$\begin{array}{c}\text { Oral conditions } \\
\text { and diseases }\end{array}$} & \multicolumn{3}{c}{$\begin{array}{c}\text { Xerostomia } \\
\text { No Patients }\end{array}$} \\
\cline { 2 - 4 } & SI & NO & $p$-Value \\
\hline Atrophic glossitis & $59 / 139$ & $11 / 68$ & $<0.0001$ \\
\hline $\begin{array}{c}\text { Denture } \\
\text { stomatitis }\end{array}$ & $27 / 140$ & $4 / 71$ & 0.005 \\
\hline
\end{tabular}

Xerostomia was defined as a score greater than 14,5 total points in the Xerostomia Inventory.

Significant associations were considered with a $p<0.05$

On multivariate logistic regression analysis, female gender odds ratio (OR=2.46, 95\% confidence interval $(\mathrm{Cl}): 1.19-5.11, \mathrm{p}=0.015)$; denture stomatitis $(\mathrm{OR}=3.71,95 \% \mathrm{Cl}: 1.03-13.3, \mathrm{p}=0.045)$ and atrophic glossitis $(\mathrm{OR}=3.72,95 \% \mathrm{Cl}: 1.78-8.1, \mathrm{p}=0.001)$ were significantly associated with hyposalivation. Only female gender $(\mathrm{OR}=2.54 ; 95 \% \mathrm{Cl}: 1.19-5.43$, $p=0.016$ ) was significantly associated with xerostomia. Comparison of age, medications, smoking habits, drinking alcohol, fissure tongue, dysgeusia and BMS do not associate with xerostomia and hyposalivation (Table 5).

\section{DISCUSSION}

This study identified the risk factors of xerostomia and hyposalivation in senior people 60 years and older, and investigated the relationship 
Table 5: Multivariate logistic regression models by forward stepwise selection of factors associated with hyposalivation and xerostomia.

\begin{tabular}{c|c|c|c|c}
\hline \multicolumn{1}{c}{ Risk Factors } & \multicolumn{1}{c}{ p-Value } & \multicolumn{1}{c}{ OR } & \multicolumn{1}{c}{$(95 \%) \mathbf{C l}$} \\
\cline { 2 - 5 } Hyposalivation & Female & 0.015 & 2.463 & {$[1.188-5.107]$} \\
\cline { 2 - 5 } & $\begin{array}{c}\text { Denture } \\
\text { Stomatitis }\end{array}$ & 0.045 & 3.709 & {$[1.031-13.340]$} \\
\cline { 2 - 5 } & $\begin{array}{c}\text { Atrophic } \\
\text { Tongue }\end{array}$ & 0.001 & 3.717 & {$[1.707-8.095]$} \\
\hline Xerostomia & Female & 0.016 & 2.542 & {$[1.190-5.428]$} \\
\hline
\end{tabular}

among medication, habits and other oral complications with these pathologies, using the spitting method and xerostomia inventory-Spanish. The hypothesis was partially supported because of there is not a statistically significant correlation between xerostomia and hyposalivation, but multivariate logistic regression analysis revealed that being a woman was significantly associated with xerostomia and hyposalivation.

The current study revealed high rates of xerostomia (81\%) compared with other studies where the prevalence varies from $17.2 \%$ to $64.8 \%$ in senior people ${ }^{(11,14,21)}$. A great prevalence of xerostomia in female was found $(84.3 \%)$, with a statistically significant difference comparing to male, this agrees with other observed findings by other authors $(3,8,9,14,17,18)$.

Regarding hyposalivation in this study, the total participants, $66.4 \%$ presented this condition, higher than the prevalence of hyposalivation registered in general population values, ranging from $11.5 \%$ to $47 \%(14,18)$ Hyposalivation was significantly associated with females. Several researches have demonstrated that these differences could be explained by hormonal changes that women experience during the menopause ${ }^{(4,16)}$.

Among 171 patients who reported having xerostomia, $69 \%$ of them had hyposalivation, these data suggest that xerostomia may occur independent of the amount of saliva secreted by patients, so it is important to perform the evaluation of both xerostomia as hyposalivation in senior population.

In the current research, most frequent systemic diseases were hypertension $(58 \%)$ and diabetes $(21 \%)$, which is in concordance with previous studies ${ }^{(23,25)}$.

Several studies have reported that most medications used in senior population provoked xerostomia and/or hyposalivation $(6-8,10,14,15,18,27)$. Unlike some authors ${ }^{(8,6)}$ who found a significant relationship between drug use and perception subjective or objective of dry mouth. The present study found a significant relationship only between xerostomia and intake anxiolytic and tricyclic antidepressants. These findings agreed with those obtained by Ohara et al. ${ }^{(14)}$ who showed that people with major depression were more likely to suffer from xerostomia, but it was no found association between depression and hyposalivation.

Another factor identified regarding dry mouth were the habits of tobacco smoking and alcohol consumption ${ }^{(15,18,23)}$. When investigating the relation of smoking and amounts of saliva, this study found a significant relationship between smoking habits and hyposalivation, like those reported in previous studies ${ }^{(3,11,18-20)}$ Whereas, other studies demonstrated that hyposalivation and xerostomia are not associated with tobacco and alcohol consumption ${ }^{(15,23)}$.

The data of multiple regression analyses in the current study show that with being female, oral candidiasis, denture stomatitis and atrophic tongue were significantly associated with hyposalivation. For xerostomia, only for being a woman was a risk factor.

It is widely stated that patients with hyposalivation and/or xerostomia, the more of the senior patients are also affected by candidiasis, dysgeusia, fissured tongue, atrophic tongue, halitosis, burning mouth syndrome, caries, periodontal diseases and taste disturbances ${ }^{(3,7,9,18,28)}$. In the present study, analysis of hyposalivation data and oral mucosal pathologies, were significantly associated with dysgeusia, fissured tongue, atrophic glossitis and denture stomatitis. By linking these oral pathologies with xerostomia, a significant relationship only with atrophic tongue, and denture stomatitis was found. This finding is in concordance with Kimori et al. ${ }^{(28)}$, who found a high Candida CFUs, low stimulated saliva flow rate and advanced age were identified as closely associated factors for the risk of development of atrophic tongue. On the other hand, Nakamura et al. ${ }^{(33)}$, reported C. albicans was associated with atrophic glossitis in xerostomia patients who had no systemic predisposing factors, indicating that $\mathrm{C}$. albicans remains a treatment target for Candida-related atrophic glossitis.

The study included some limitations. The patient's recruitment was performed in a dental clinic of a tertiary care center. The study did not include the measure of patient's quality life outcomes, and saliva composition profile study.

Dentists should be aware of the symptoms of xerostomia and hyposalivation in senior patients (60 years older). They should administer proper treatment to prevent the development of oral lesion. It is important to carry out future research on the prevalence of hyposalivation in the Senior Chilean population. As well as to include routine unstimulated whole-salivary flow measurement, as part of the clinical examination for a correct design of the treatment plans, achieving to improve their quality of life.

\section{CONCLUSIONS}

1. No statistically significant association was found between hyposalivation and xerostomia.

2. Hyposalivation was a risk factor for denture stomatitis and atrophic tongue.

3. Being woman was a risk factor for xerostomia and hyposalivation.

\section{ACKNOWLEDGEMENTS}

We thank all patients for their perseverance in attending to all their controls.

\section{CONFLICT OF INTEREST STATEMENT}

None declared. Supported by the Oral Prosthetic Rehabilitation Program, School of Dentistry, Universidad de Concepción.

\section{References}

1. World Health Organization. Active ageing: a policy framework. Geneva, Switzerland: World Health Organization. 2002.

2. Minsal Chile. Programa nacional de salud de las personas adultas mayores. 2014.

3. Atkinson JC, Grisius M, Massey W. Salivary hypofunction and xerostomia: diagnosis and treatment. Dent Clin North Am. 2005;49:309-26.

4. Eliasson L, Carlen A, Laine M, Birkhed D. Minor gland and whole saliva in postmenopausal women using a low potency estrogen (estriol). Arch Oral Biol. 2003;48:511-7

5. Wiener C, Crout R, Wiener M, Plassman B, Kao E, McNeil D. Hyposalivation and xerostomia in dentate older adults. J Am Dent Assoc. 2010;141:279-84

6. Gupta A, Epstein JB, Sroussi H. Hyposalivation in elderly patients. J Can Dent Assoc. 2006;72:841-6.

7. Nagler RM, Hershkovich O. Relationships between age, drugs, oral sensorial complaints and salivary profile. Arch Oral Biol. 2005;50:7-16.

8. Guggenheimer J, Moore PA. Xerostomia: etiology, recognition and treatment. J Am Dent Assoc. 2003;143:61-9.

9. Anil S, Vellappally S, Hashem M, Preethanath R, Patil S, Samaranayake LP. Xerostomia in geriatric patients: a burgeoning global concern. J Investig Clin Dent. 2014;5:1-8.

10. Nagler RM. Salivary glands and the aging process: mechanistic aspects, healthstatus and medicinal-efficacy monitoring. Biogerontology. 2004;5:223-33.

11. Orellana MF, Lagravère MO, Boyahuk DG, Major PW, Flores-Mir C. Prevalence

of xerostomia in population-based samples: a systematic review. J Public Health
Dent. 2006;66:152-8

12. Humphrey SP, Williamson RT. A review of saliva: Normal composition, flow, and function. J Prosthet Dent. 2001:85:162-9.

13. Silvestre-Donat FJ, Miralles-Jordá L, Martinez-Mihi V. Protocol for the clinical management of dry mouth. Med Oral. 2004;9:273-9.

14. Ohara Y, Hirano H, Hideyo Y, Obuchi S, et al. Prevalence and factors associated with xerostomia and hyposalivation among community-dwelling older people in Japan. Gerodontology. 2016;33:20-7

15. Smidt D, Torpet LA, Nauntofte B, Heegaard KM, Pedersen AM. Associations between labial and whole salivary flow rates, systemic diseases and medications in a sample of older people. Community Dent Oral Epidemiol. 2010;38:422-35.

16. Laine M, Leimola-Virtanen R. Effect of hormone replacement therapy on salivary flow rate, buffer effect and $\mathrm{pH}$ on premenopausal and postmenopausal women. Arch Oral Biol.1996;41:91-6.

17. Hsu KJ, Lee HE, Lan SJ, Huang ST, Chen CM, Yen YY. Evaluation of a self-assessed screening test for masticatory ability of Taiwanese older adults. Gerodontology. 2012;29:1113-20.

18. Samnieng P, Ueno M, Shinada K, Zaitsu T, Wright FA, Kawaguchi Y. Association of hyposalivation with oral function, nutrition and oral health in community-dwelling elderly Thai. Community Dent Health. 2012;29:117-23

19. Almeida Leite C, Francis Galera M, Martínez Espinosa M, de Teles PR. Prevalence of hyposalivation in patients with systemic lupus erythematosus in Brazilian subpopulation. Int J Rheumatol. 2015; 2015: 1-6.

20. Maeshima E, Furukawa K, Maeshima S, Kohiba H, Sakamoto W. Hyposalivation 
in autoimmune diseases. Int J Rheumatol. 2013;33:3079-82.

21. Thomson WM, Chalmers JM, Spencer AJ, Williams SM. The xerostomia inventory: a multi-item approach to measuring dry mouth. Community Dent Health. 1999;16:12-17

22. Kakoei S, Haghdoost AA, Rad M, Mohammadalizadeh S, et al. Xerostomia after radiotherapy and its effect on quality of life in head and neck cancer patients. Arch Iran Med. 2012;15:214-8.

23. Vila A, Abati S. Risk factors and symptoms associated with xerostomia: a crosssectional study. Aust Dent J. 2011:56:290-5.

24. Imanguli MM, Atkinson JC, Mitchell SA, Avila DN, et al. Salivary glands involvement by chronic graft- versus-host disease: prevalence, clinical significance and recommendations for evaluation. Biol Blood Marrow Transplant. 2010;16:1362-9. 25. Habbab KM, Moles DR, Porter SR. Potential oral manifestations of cardiovascular drugs. Oral Dis. 2010;16:769-73.

26. Singh ML, Papas A. Oral implications of polypharmacy in the elderly. Dent Clin North Am. 2014;58:783-96

27. Sato I, Akazawa M. Polypharmacy and adverse drug reactions in Japanese elderly taking antihypertensives: a retrospective database study. Drug Health Patient Saf. 2013;5:143-50.

28. Kimori H, Yamamoto K, Yamachika S, Tsurumoto A, et al. Factors associated with the presence of atrophic tongue in patients with dry mouth. Gerontology. 2015;32(1):13-17.

29. World Health Organization. Oral health survey. Basic methods, 5th ed. Geneva: 2013; 53-54

30. Serrano C, Fariña MP, Pérez C, Fernández M, Forman K, Carrasco M. Translation and validation of a Spanish version of the xerostomia inventory. Gerodontology. 2016; 33:506-12.

31. Navezesh M. How can oral health care providers determine if patients have dry mouth? J Am Dent Assoc. 2003;134:613-17.

32. Sreebny LM, Schwartz SS. A reference guide to drugs and dry mouth-2nd edition. Gerodontology. 1997;14:33-47.

33. Nakamura S, Okamoto MR, Yamamoto K, Tsurumoto A, Yoshiro Y, Iwabuchi $\mathrm{H}$, et al. The Candida species that are important for the development of atrophic glossitis in xerostomia patients. BMC Oral Health. 2017;17:153. 